\title{
Sectores estratégicos para el cambio de la Matriz Productiva: perspectiva de la ciudadanía y de los empresarios de Tungurahua
}

\author{
URL: http://revistas.uta.edu.ec/erevista/index.php/bcoyu/article/view/687 DOI: http://dx.doi.org/10.31164/bcoyu.20.2019.687
}

\section{Lissette Flores-Cevallos'; Carlos Flores-Tapia²; Giovanna Cuesta-Chávez³; Amparito Pérez- Barrionuevo ${ }^{4}$}

Fecha de recepción: 29 de enero de 2019

Fecha de aceptación: 15 de febrero de 2019

\section{Resumen}

Ecuador es un país primario exportador de materias primas que otros países agregan valor mediante procesos industriales e incorporación de servicios, desaprovechando de esta manera las posibilidades de incrementar los ingresos. Por ello, el cambio de la matriz productiva representa un reto y una oportunidad para la economía ecuatoriana. En tal sentido, el gobierno nacional priorizó 14 sectores productivos para optimizar la inversión. Ahora bien, este estudio, mediante la aplicación de encuestas tabuladas y procesadas estadísticamente, buscó determinar si dicha priorización responde o no a las expectativas e intereses de la ciudadanía y del empresariado de la provincia de Tungurahua.

Palabras claves: Economía, productividad, competitividad, desarrollo territorial.

\section{Abstract}

Ecuador is a primary exporter of raw materials that other countries add value through industrial processes and incorporation of services, thus squandering the possibilities of increasing revenues. Therefore, the change in the productive matrix represents a challenge and an opportunity for the Ecuadorian economy. In this regard, the national government prioritized 14 productive sectors to optimize investment. Now, this study, through the application of tabulated and statistically processed surveys, sought to determine whether or not this prioritization responds to the expectations and interests of the citizens and the business community of the province of Tungurahua.

Keywords: Economy, productivity, competitiveness, territorial development.

\section{Introducción}

En la última década, en el Ecuador ha tomado auge la matriz productiva para desarrollar nuevas empresas que puedan sustituir importaciones. En este sentido, la actividad productiva de Tungurahua tiene como ejes principales:

- La producción agropecuaria y, dentro de esta, la agricultura familiar campesina (AFC) y la producción pecuaria especialmente de leche y carne

La producción manufacturera en la elaboración de varios productos exclusivamente para consumo nacional.

- El turismo, para aprovechar los elementos naturales y culturales urbanos y rurales. (Dirección de Planificación, 2015)

Al respecto, según datos proporcionados por el Instituto Nacional de Estadística y Censos (INEC, 2017), en Tungurahua existen 3.480 personas naturales y 150 personas jurídicas dedicadas a la industria manufacturera, de las cuales: 3.194 son microempresas, 357 pequeñas empresas, 61 medianas empresas, y 18 grandes empresas, evidenciándose que la provincia de Tungurahua tiene el 33,3\% de mayor índice de ocupación plena. De esta manera, el estudio tuvo como objetivo general: identificar los sectores que deben priorizarse para el impulso de la economía de la provincia. Con lo cual, se facilita atender a las organizaciones que desarrollan producciones para la sustitución de importaciones en la provincia, dado que puede apaliarse con ello, la falta de empleo y las limitaciones de calidad de vida de los ciudadanos.

La Matriz Productiva permite desarrollar determinados sectores de la economía ecuatoriana con el fin de superar el modelo primario de producción. Razón por la cual se priorizan sectores o ramas económicas del país, que puedan ser impulsadas por las empresas y los territorios subnacionales, con el objetivo fundamental de impulsar la producción y los servicios con mayor valor agregado (Flores \& Flores, 2016).

Con relación a la matriz productiva, SENPLADES (2012), menciona que:

"La forma cómo se organiza la sociedad para producir determinados bienes y servicios no se limita únicamente a los procesos estrictamente técnicos o económicos, sino que también tiene que ver con todo el conjunto de interacciones entre los distintos actores sociales que utilizan los recursos que tienen a su disposición para llevar adelante las actividades productivas. A ese conjunto, que incluye los productos, los procesos productivos y las relaciones resultantes de esos procesos, denominamos matriz productiva" (p. 7).

En tal sentido, se considera importante integrar al proyecto de la matriz productiva a los actores privados, sean familiares o individuales para impulsar determinados proyectos financiados o cofinanciados por el Estado o de manera compartida como capital mixto.

Asimismo, Escobar (2016) señala que "el cambio de matriz productiva constituye una reedición modificada de las políticas de industrialización sustitutiva de importaciones (ISI), basado en los estudios del intercambio desigual y la teoría de la dependencia" (p.29). Con lo cual, todo cambio en la economía de un país se sustenta en métodos, normativas, regulaciones, políticas y técnicas, que apuntan a la aplicación de procesos de transformación significativa, mediante la: motivación e incentivos a pequeños productores, fuentes de financiamiento productivo, capacitación, infraestructura y la participación decidida de los actores de la economía.

En tal sentido, se considera que no solo el apoyo de las instituciones permite lograr la adopción e implementación de la matriz productiva, pues existen factores que se requieren para modificar la matriz productiva como base de un estado plurinacional e intercultural (Figura 1).

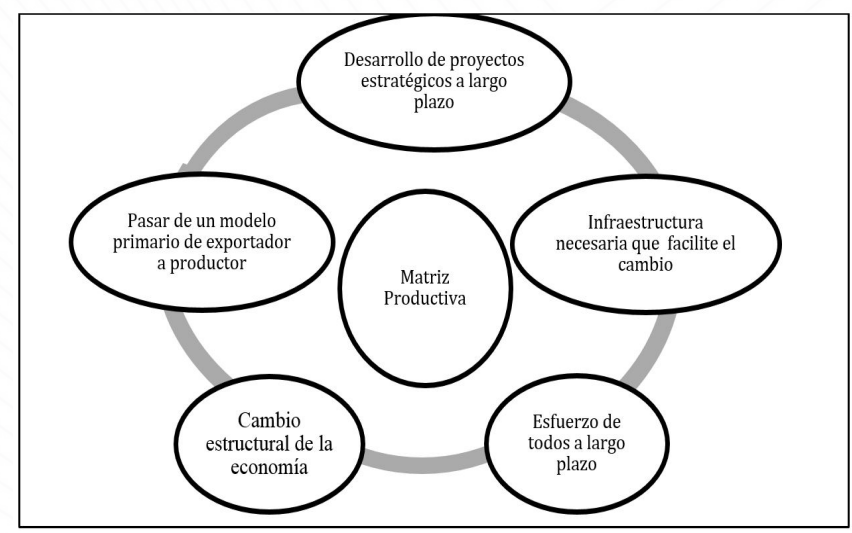

Fuente: Elaboración propia a partir de Secretaría Nacional de Planificación y Desarrollo (2012) Figura 1. Factores que inciden en el cambio de la matriz productiva

En consecuencia, se establecen cuatro ejes (Banco Central del Ecuador, 2013) para cambiar el patrón de especialización primario-exportador a un patrón de exportador de conocimientos, servicios y productos con valor agregado (Figura 2).

'Fundación Los Andes. Investigadora Directora Ejecutiva. Ambato-Ecuador. E-Mail: lisceva94@gmail.com. ORCID: https://orcid.org/0000-0002-9370-217X

2Pontificia Universidad Católica del Ecuador. Docentelnvestigador de la Escuela de Administración de Empresas. Ambato-Ecuador. E-Mall: florestapiacarlos@yahoo.com. ORCID: https://orcid.org/0000-0002-1892-6309 ${ }^{3}$ Universidad Técnica de Ambato. Docente de la Facultad de Contabilidad y Auditoría. Ambato-Ecuador. E-Mail: ga.cuesta@uta.edu.ec. ORCID: https://orcid.org/0000-0002-0626-9196

"Pontificia Universidad Católica del Ecuador. Docente de la Escuela de Administración de Empresas. Ambato-Ecuador. E-Mail: aperez@pucesa.edu.ec. 
En consecuencia, se establecen cuatro ejes (Banco Central del Ecuador, 2013) para cambiar el patrón de especialización primario-exportador a un patrón de exportador de conocimientos, servicios y productos con valor agregado (Figura 2).

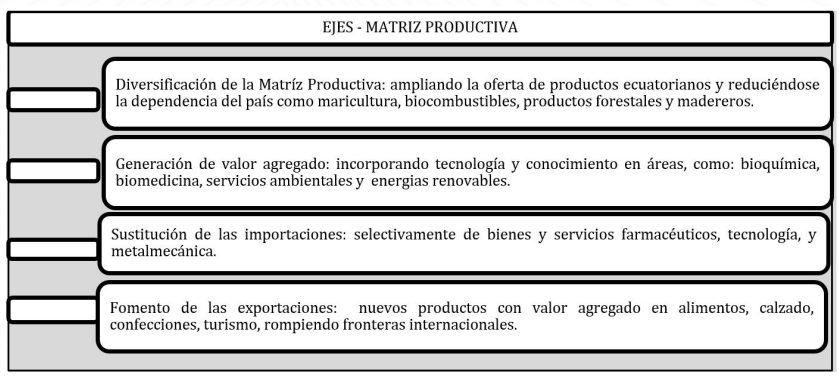

Fuente: Elaboración propia a partir de Banco Central del Ecuador (2013)

Figura 2. Ejes para la transformación de la matriz productiva

Como resultado del proceso de cambio, motivación e incentivos se tienen ejemplos de algunos sectores que se han ido desarrollando con un enfoque productivo, destacando que las exportaciones no petroleras de enero a noviembre de 2018 fueron de USD 11.675,0 millones, superando a las exportaciones petroleras que fueron de USD. 8.225,2 millones; siendo que el monto de exportaciones no petroleras superior en 5,3\% (USD 585,8 millones) con respecto al periodo enero-noviembre de 2017 (Asobanca, 2019; BCE, 2019). Por su parte, el porcentaje de las exportaciones de los productos tradicionales con respecto al total de exportaciones, durante el período enero- noviembre de 2017, según el BCE (2019) fue el siguiente: camarón (25,5\%); plátano $(24,8 \%)$; cacao y elaborados $(5,8 \%)$; atún y pescado $(2,4 \%) ;$ y, café y elaborados $(0,6 \%)$, en las no tradicionales destacan: enlatados de pescado $(8,9 \%)$, flores naturales $(6,8 \%)$, otras manufacturas de metal $(3,3 \%)$, extractos y aceites vegetales $(2,0 \%)$, productos mineros $(2,1 \%)$, madera $(1,9 \%)$, elaborados de banano $(1,3 \%)$, manufacturas de cuero, plástico y caucho $(1,2 \%)$, químicos y fármacos $(1,2 \%)$, vehículo $(0,8 \%)$, jugos y conservas de frutas $(0,8 \%)$; el resto de productos representan el (10,6 \%). Por ello, es necesario dejar por sentado que el cambio de la matriz productiva como política de Estado depende del dinamismo de la economía y del grado de participación o empoderamiento de los principales actores económicos del país.

\section{Metodología}

La metodología de la investigación fue de tipo descriptivo-explicativo, con un enfoque mixto (cuantitativo-cualitativo). Se aplicó el método de análisis-síntesis para examinar con un alto nivel de detalle el comportamiento de la matriz productiva en Tungurahua.

Asimismo, se profundizó en el análisis de la percepción de los sectores prioritarios por parte de la ciudadanía y del sector empresarial, para lo cual se aplicó encuestas tanto a la ciudadanía y al sector empresarial, abarcándose los nueve cantones de la provincia de Tungurahua. Asimismo, las fuentes de información primarias se analizaron en dos escenarios como se muestra a continuación.

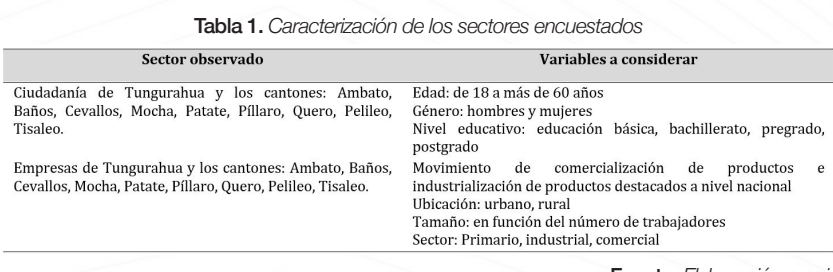

Fuente: Elaboración propia

Como se consideraron dos ejes fundamentales para la observación de resultados, se determinó de igual manera la población y la muestra combinada para los objetos de estudio: conglomerados y estratos para el sector ciudadanía y por conglomerados para el sector empresarial, considerando los siguientes supuestos:

- Error estadístico del 5\%.

- Nivel de Confianza 95\%.
- Desviación de la población al cuadrado o varianza 0,25 porque se trabaja en el centro, es decir 0,5 de éxito y 0,5 de fracaso.

- Valor tipificado "z", que corresponde a 1,96 doble cola.

- Población sector ciudadanía: 504.583 habitantes (INEC, 2018).

- Población sector empresarial: 43.036 empresas (INEC, 2018).

El cálculo del tamaño de la muestra para el sector ciudadanía resultó en 384 unidades a encuestar, tomando muestras proporcionales a la población por cantones y por género (hombres, mujeres) y de 381 empresas del sector empresarial, tomando muestras proporcionales por tipo de empresa (sector primario, secundario y terciario). Luego, se procedió con el análisis de los resultados de la investigación, los mismos que se presentan a continuación.

\section{Resultados}

\section{Sector ciudadanía}

En la Figura 3 se muestra que el $86 \%$ de la ciudadania menciona que el sector más representativo y con mayor impacto productivo es el sector de los alimentos frescos y procesados, sequido del sector de Confecciones textiles y cuero y calzado; la construcción (80\%), Turismo (77\%) y Biotecnología (76\%). Evidencíandose un alto nivel de coincidencia entre la priorización del gobierno nacional y la priorización de los sectores estratégicos desde la perspectiva de la ciudadanía tungurahuense. Considerando que el cambio de la matriz productiva implica que el país pase de un modelo productivo primario a uno industrial y de servicios.

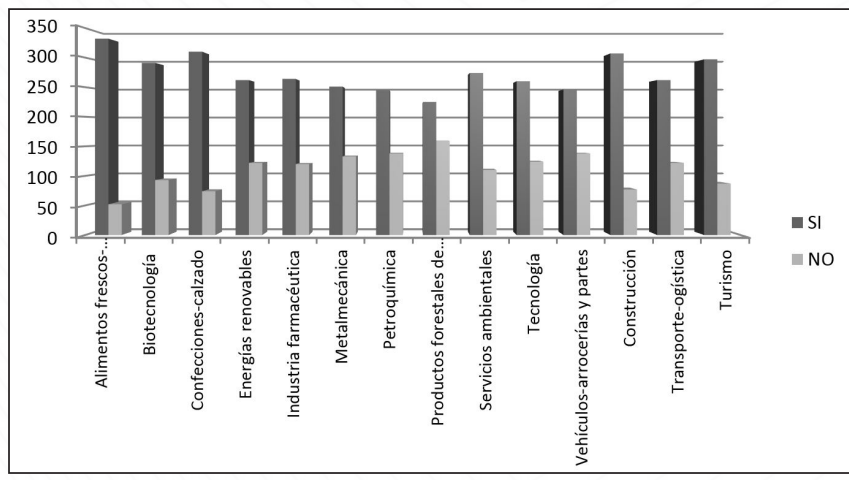

Fuente: Elaboración propia a partir de la encuesta aplicada a la población de Tungurahua - 2018 Figura 3. Priorización de los sectores estratégicos - ciudadanía

A continuación, en la Figura 4 se presenta la priorización del sector alimentos frescos, grupo en el cual se destacan cultivo de banano-café-cacao con un $86 \%$, seguido del sector dedicado a la elaboración de productos lácteos (81\%), en relación al cultivo de cereales (79\%), elaboración de chocolate, productos de confitería, otros productos alimenticios con el (78\%), acuicultura y pesca de camarón (77\%), elaboración de productos de la molinería, panadería y fideos (76\%), azúcar (73\%), procesamiento y conservación de camarón, procesamiento y conservación de pescado y otros productos acuáticos (71\%), pesca y acuicultura70\% (excepto de camarón), procesamiento y conservación de carne (69\%), finalmente, la elaboración de bebidas y productos de tabaco (51\%).

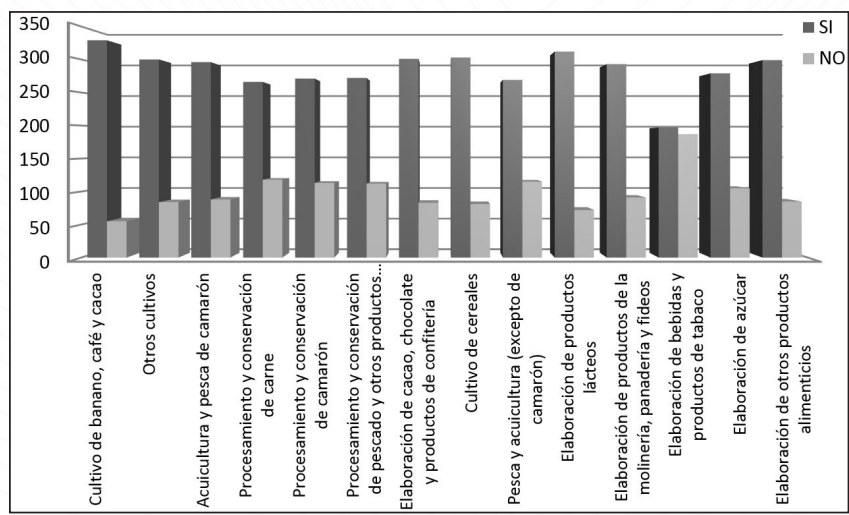

Fuente: Elaboración propia a partir de la encuesta aplicada a la población de Tungurahua - 2018 Figura 4. Priorización de productos del sector alimentos frescos y procesados - ciudadanía 
Con el objetivo de impulsar el cambio de la matriz productiva, la Figura 5 muestra que el $87 \%$ recomiendan promover el cultivo de flores, seguido de la cría de animales, explotación de minas y canteras, fabricación de productos del caucho y plástico, metales comunes, maquinaria y equipo, hogares privados con servicio doméstico, productos derivados del metal, comercio al por mayor y al por menor, actividades de servicios financieros, inmobiliarias, actividades profesionales, técnicas y administrativas, administración pública, defensa; planes de seguridad social obligatoria, servicios sociales y de salud, fabricación de muebles, artesanía, reparación de vehículos automotores y motocicletas, entre otros.

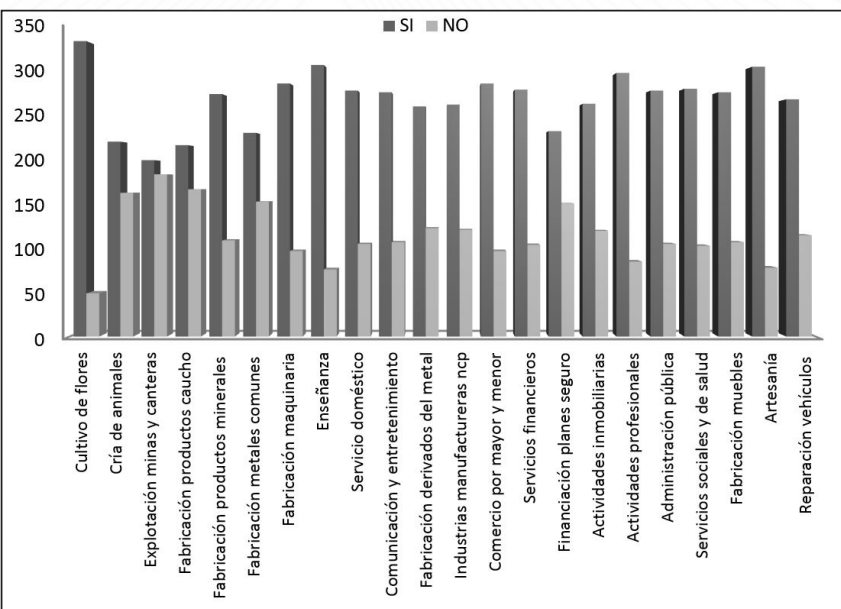

Fuente: Elaboración propia a partir de la encuesta aplicada a la población de Tungurahua - 2018 Figura 5. Sectores que también deben priorizarse para el cambio de la matriz productiva - ciudadanía

\section{Análisis e interpretación de resultados - Ciudadanía}

En base a los resultados obtenidos de la encuesta realizada, se aprecia que existe un interés favorable de la ciudadanía en la priorización del sector estratégico de alimentos frescos y procesados como parte del cambio en la matriz productiva impulsado por el gobierno nacional. Dentro de este sector estratégico, se observa que la ciudadanía aún percibe que productos tradicionales como el cultivo de banano, café, cacao y cereales, pueden formar parte de la matriz productiva; sin embargo, un alto porcentaje de encuestados opinan que el cultivo de flores, las artesanías y el comercio al por mayor y al por menor, también deben tomarse en cuenta para lograr este propósito por su fortaleza en los altos niveles de producción.

Por otra parte, el estudio evidenció, desde la perspectiva de la ciudadanía, que los principales actores que impulsan el cambio en la matriz productiva son: el gobierno nacional, los gobiernos autónomos descentralizados y los gremios de producción. No obstante, existe un porcentaje minoritario que considera que las iglesias, los militares, los medios de comunicación social y el sistema financiero contribuyen de manera favorable al cambio de la matriz productiva.

\section{Sector Empresarial}

El sector empresarial frente a la pregunta: ¿Está de acuerdo o no con la priorización de cada uno de los siguientes sectores estratégicos realizada por el Gobierno Nacional con el fin de impulsar el cambio de la matriz productiva?, jerarquizan el apoyo principalmente en los sectores relacionados con el turismo, productos forestales de madera, tecnología (software, hardware y servicios informáticos), transporte y logística, alimentos frescos y procesados, confección de calzado, construcción, servicios ambientales y vehículos, automotores, carrocerías y partes, energías renovables, industria farmacéutica, petroquímica, y en los últimos lugares metalmecánica con y biotecnología -bioquímica y biomedicina- (Figura 6).

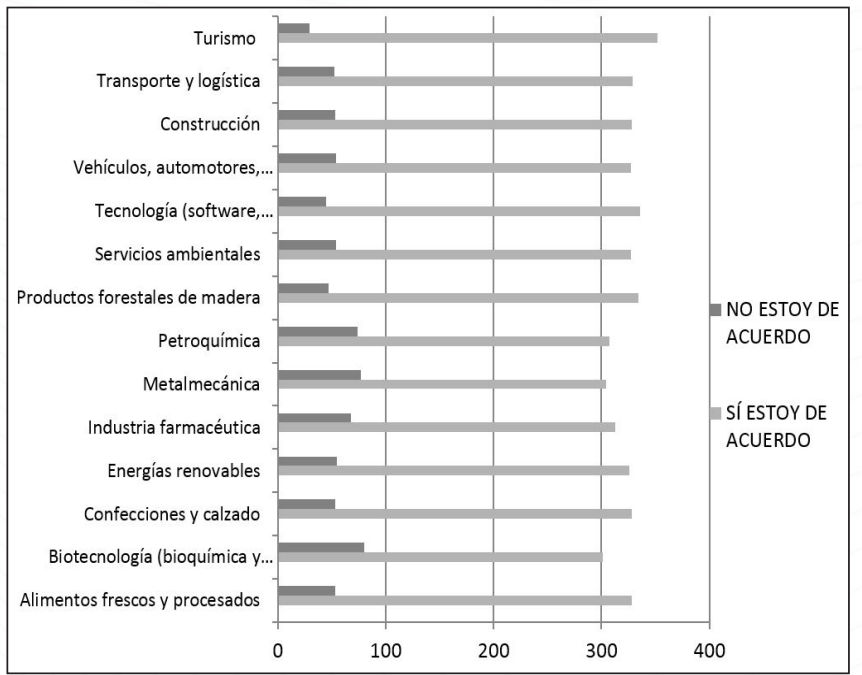

Fuente: Elaboración propia a partir de la encuesta aplicada al sector empresarial de Tungurahua - 2018 Figura 6. Priorización de los sectores estratégicos - sector empresarial

En relación a la priorización del sector Alimentos frescos y procesados, se observa en la Figura 7 que los empresarios consideran necesario apoyar principalmente a los subsectores banano-café cacao, lácteos, otros productos alimenticios, cereales, elaboración de cacao, chocolate y confitería (notándose que algunos de estos productos tienen el apoyo del sector empresarial para su priorización, a pesar de que no se cultivan en el territorio de la provincia de Tungurahua); y en menor medida, se inclinan por elaboración de bebidas y tabaco, elaboración de azúcar, procesamiento y conservación de carne, entre otros.

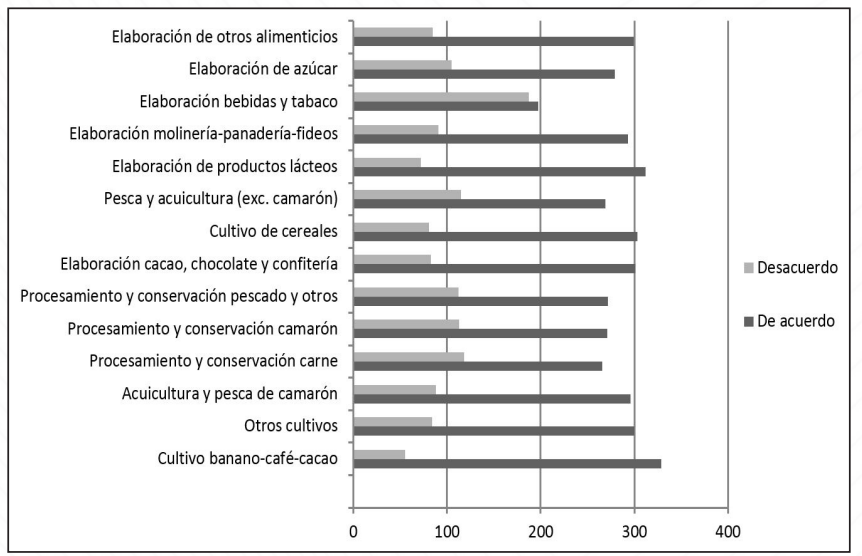

Fuente: Elaboración propia a partir de la encuesta aplicada al sector empresarial de Tungurahua - 2018 Figura 7. Priorización de productos del sector alimentos frescos y procesados -sector empresarial

De los datos observados, en la Figura 8 se determina que los sectores que también se deben promover son: cultivo de flores, la elaboración de artesanías, la enseñanza, las actividades profesionales, comercio al por mayor y menor, actividades de servicios financieros, fabricación de muebles, servicios financieros, servicios sociales y de salud, actividades inmobiliarias, comunicación y entretenimiento, fabricación de maquinaria y equipo, fabricación de otros productos minerales no metálicos, fabricación de productos derivados del metal, entre otros. 


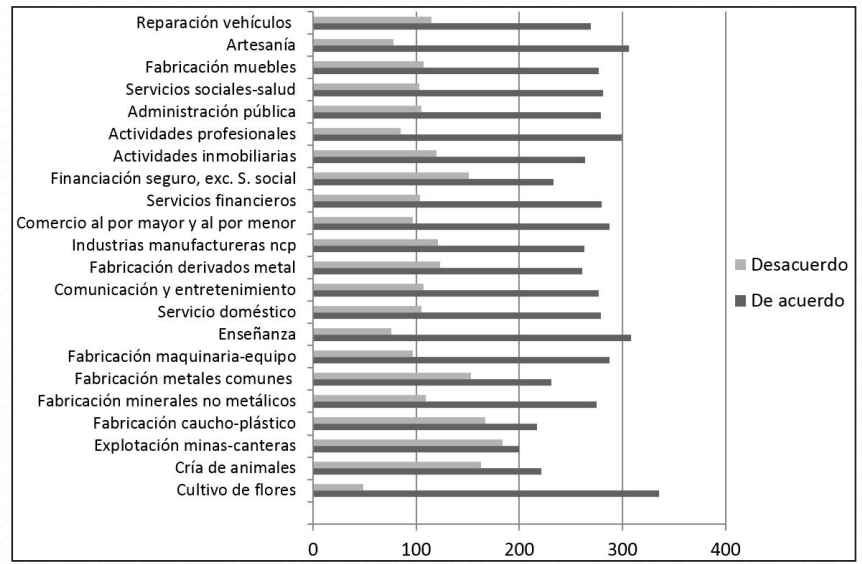

Fuente: Elaboración propia a partir de la encuesta aplicada al sector empresarial de Tungurahua - 2018 Figura 8. Sectores que también deben priorizarse para el cambio de la matriz productiva - sector empresanial

\section{Análisis e interpretación de resultados - empresas}

De los datos obtenidos, una vez realizada la encuesta a los empresarios de la provincia de Tungurahua, se corrobora que es conveniente el cambio de matriz productiva de manera urgente, si se quiere dejar atrás el modelo primario exportador y adoptar un modelo exportador de conocimientos, servicios y productos con valor agregado. En este sentido, es necesario el fortalecimiento de los sectores endógenos, como es el caso del sector turístico, dadas las condicionales naturales propias del país, particularmente en la provincia de Tungurahua.

Adicionalmente, desde la perspectiva del sector empresarial, el Gobierno Nacional es el principal impulsor del cambio de la matriz productiva, seguido de los gobiernos autónomos descentralizados (prefecturas/ municipios/Juntas Parroquiales), y en menor medida militares, ciudadanía, academia, estudiantes e iglesias.

\section{Conclusiones}

La ciudadanía y el sector empresarial de la provincia de Tungurahua coinciden con la necesidad de impulsar los sectores estratégicos priorizados por el gobierno nacional para el cambio de la matriz productiva. Adicionalmente, se evidencia que el sector empresarial está mucho más de acuerdo con la priorización del gobierno nacional que la ciudadanía tungurahuense. Sin embargo, también es cierto que se requiere incluir en la priorización otros sectores productivos que respondan a las especificidades territoriales, como por ejemplo -desde la perspectiva tungurahuense- el cultivo de flores, la elaboración de artesanías, la enseñanza, las actividades profesionales, comercio al por mayor y menor, actividades de servicios financieros, fabricación de metales comunes y de productos derivados del metal, entretenimiento, recreación y otras actividades de servicios, entre otros.

De los resultados obtenidos en el estudio, también puede concluirse que la ciudadanía tungurahuense no está tan consciente de la necesidad del cambio de la matriz productiva como lo está el sector empresarial, porque aún prioriza el cultivo de productos primarios de exportación como banano-café-cacao, flores, otros productos alimenticios, cereales; mientras que en el sector empresarial, en cambio, ya se plantea el impulso al sector turismo y a otros relacionados con la industria manufacturera y de desarrollo tecnológico.

Por otra parte, es necesario considerar el nivel de aportación y articulación al crecimiento y desarrollo económico de la provincia de Tungurahua de los sectores económicos priorizados y del aprovechamiento de las oportunidades nacionales e internacionales, lo que permitirá la identificación de proyectos y negocios que contribuyan a mejorar la competitividad territorial, y por ende,al buen vivir de sus ciudadanos y ciudadanas.
Ahora bien, los resultados del estudio muestran que de los 14 sectores productivos considerados para el cambio de la matriz productiva, los más importantes son comercio al por mayor y menor, alimentos frescos y procesados, cuero y calzado, carrocerías, construcción, entre otros. Asimismo, se recomienda no sólo desarrollar los sectores productivos de manera independiente, sino que es necesario integrarlos en cadenas productivas y clústeres; de tal manera que se optimicen los recursos y se obtengan sinergias significativas que apuntalen el desarrollo económico y social, del Ecuador, en general, y de la provincia de Tungurahua, en particular.

La priorización de los sectores que también se deben impulsar para el cambio de la matriz productiva, desde la perspectiva de la ciudadanía y del sector empresarial de Tungurahua, evidencia la necesidad de la articulación de la política de transformación productiva de los territorios subnacionales con la política nacional y viceversa, porque si solo se toma en cuenta la política nacional se puede correr el riesgo de dejar de lado capacidades y potencialidades endógenas del sistema productivo de los territorios subnacionales, desaprovechando oportunidades de vertebración y encadenamientos productivos interterritoriales y nacionales.

Dicho lo anterior, resulta importante que los sectores priorizados como impulsores del cambio de la matriz productiva sean incorporados en los planes de desarrollo nacional y en los planes de desarrollo de los territorios subnacionales, de esa manera pueden optimizarse los recursos locales y nacionales, tanto públicos como privados, que se asignen al fomento productivo y a la mejora de la competitividad.

Finalmente, una de las líneas de estudio a futuro que se deriva de esta investigación puede ser la identificación y análisis de la correspondencia entre la priorización de los sectores estratégicos para el cambio de la matriz productiva del gobierno nacional con la identificación de sectores clave realizada aplicando, por ejemplo el método Rasmussen, a partir de la Matriz Insumo Producto nacional. Otra línea de investigación puede ser el estudio de las capacidades de vertebración y encadenamientos productivos interterritoriales y regionales que pueden establecerse a partir de la priorización de los sectores estratégicos para el cambio de la matriz productiva.

\section{Referencias}

Asobanca. (enero de 2019). Boletín Macroeconómico. Obtenido de: $\quad$ https://www.asobanca.org.ec/publicaciones/bolet\%C3\%ADn-macroecon\%C3\%B3mico/bolet\%C3\%ADn-macroecon\%C3\%B3mico-enero-2019

Banco Central, E. (29 de junio de 2013). Cuatro ejes rigen la Matriz productiva. Obtenido de http://biblioteca.bce.ec/cgi-bin/koha/opac-detail.pl?biblionumber $=104765 \#$

BCE. (2018). Cuentas provinciales - Banco Central del Ecuador. Retrieved from https://www.bce.fin.ec/index.php/component/k2/item/763-cuentas-nacionales

BCE. (2019). Información Estadística - Banco Central del Ecuador. Retrieved from https://contenido.bce.fin.ec/home1/estadisticas/bolmensual/IEMensual.jsp

Buen Vivir , P. (2010). Estrategia Territorial Nacional - ETN. Obtenido de https://www.google.com.ec/url?sa=t\&rct=j\&q=\&esrc=s\& source $=$ web \&cd $=5 \& c a d=r j a \& u a c t=8 \& v e d=0 a h U K E w i 2 h K n 1 p s D U A h-$ VHMyYKHVvIBhkQFgg4MAQ\&url=http\%3A\%2F\%2Fwww. buenvivir.gob. ec\%2Fdocuments\%2F10157\%2Fa77659d2-a53b-4905-8d40-65d3cf4f6db3\&usg=AFQjCNHsPLgVj3 5 Vkdg6CZM5CS

CEPAL. (2010). Anuario Estadístico de América Latina y el Caribe 2009. Mexico: CEPAL

Chiriboga, M. \& Wallis, B. (Noviembre de 2010). Diagnóstico de la pobreza rural en ecuador y respuestas de política pública. Obtenido de http://www. rimisp.org/wp-content/files_mf/1366317392Diagnosti...pdf

Daza, E. (2015). Estado y transformación productiva en el campo Ecuador 2013/2014. Revista GeoNordeste, 274-294.

Dirección de Planificación. (2015). Agenda Tungurahua 2015-2017. Recuperado el 4 de agosto de 2017, de https://issuu.com/gobtungurahua/ docs/pdf_agenda_tungurahua_2015-2017_hgp 
Ecuador, B. C. (Mayo de 2017). Evolución de la Balanza Comercial enero marzo 2017. Obtenido de Subgerencia de Programación y Regulación Dirección Nacional de Síntesis Macroeconómica: https://contenido.bce.fin. ec/documentos/Estadisticas/SectorExterno/BalanzaPagos/balanzaComercial/ ebc201705.pdf

Escobar Jiménez, C. (s.f.). Análisis de la política ecuatoriana de becas de estudios de posgrado en el exterior y su relacion con el cambio de matriz productiva. Revista Latinoamericana de Políticas y Acción Pública, 23-49.

Espinosa, R. (2015). Sistema Nacional de Informaciòn- SIM. Obtenido de Ministerio Coordinador de Produccion, empleo y Competitividad : http://www. produccion.gob.ec/ministro-richard-espinosa-inauguro-expoalimentar-2015/

Espinosa, R. (24 de mayo de 2014). El ciudadano. Obtenido de http://www.elciudadano.gob.ec/ministro-espinoza-cambio-de-matriz-productiva-requiere-de-la-participacion-del-sector-publico-y-privado/

Flores-Tapia, C. \& Flores-Cevallos, L. (2016). Herramientas para simulación de matrices productivas óptimas del Ecuador. Economia y Administraciòn , 7(2), 5 .

Gachet, N. (2017). La Matriz Productiva. la Repùblica, 4-6.

INEC. (junio de 2013). Directorio de empresas 2015. Obtenido de http:// www.ecuadorencifras.gob.ec/directoriodeempresas/

Izurieta, N. P. (2015). El Ecuador y el Proceso de Cambio de la Matriz Productiva: Consideraciones para el desarrollo y equilibrio de la Balanza Comercial. Observatorio de la Economia Latinoamericana reviste eumednet, 10.

Matute, A., Mora, ,. J., \& Mora, R. (2017). Ventajas y desventajas del cambio de la matriz productiva y su incidencia en el sector cafetero de la provincia de El Oro. Tecnológica ESPOL, 4-10.

Ministerio de coordinacion de la Producciòn, e. y. (mAYO de 2011). Agendas para la transformacion productiva territorial: Provincia de Tunguahua. Obtenido de http://www.produccion.gob.ec/wp-content/uploads/downloads/2013/O2/AGENDA-TERRITORIAL-TUNGURAHUA.pdf

Palacios, \& Reyes. (2016). Cambio de la matriz productiva del Ecuador y su efecto en el comercio exterior. Dialnet Plus, 418-431.

Prebisch, R. (2012). El desarrollo económico de la América Latina y algunos de sus principales problemas. Mexico: CEPAL en Santiago (Estudios e investigaciones).

BCE. (2018). Cuentas provinciales - Banco Central del Ecuador. Retrieved from https://www.bce.fin.ec/index.php/component/k2/item/763-cuentas-nacionales

INEC. (2018). Ecuador en cifras. Retrieved from http://www.ecuadorencifras.gob.ec

Secretaría Nacional de Planificación y Desarrollo. (2012). Transformación de la Matriz Productiva. SENPLADES. Retrieved from http://www.planificacion. gob.ec/wp-content/uploads/downloads/2013/01/matriz_productiva_WEBtodo. $p d f$

SENPLADES. (MAYO de 2013). Plan Nacional del Buen Vivir. Obtenido de http://www.buenvivir.gob.ec/69

SENPLADES. (2014). Agenda regulatoria para la transformaciòn productiva. Quito: Secretaria Nacional de Planificacion y Desarrollo .

SENPLADES. (18 de Marzo de 2014). Estrategia Territorial EcuatorianaETE. Obtenido de www.buenvivir.gob.ec/documents/10157/a77659d2-a53b4905-8d40-65d3cf4f6db3

SENPLADES. (2015). Agenda Zonal - Zona 3 Centro - Provincias de Cotopaxi,Tungurahua, Chimborazo y Pastaza 2013-2017. Obtenido de http:// www.planificacion.gob.ec/wp-content/uploads/downloads/2015/11/Agenda-zona-3.pdf

SENPLADES. (ENERO de 2016). Secretaria Nacional de Planificaciòn y Desarrollo. Obtenido de http://www.planificacion.gob.ec/wp-content/uploads/ downloads/2016/01/9RC.pdt

SENPLADES. (Octubre de 2012). Folleto informativo Proceso de desconcentración del Ejecutivo en los niveles administrativos de planificación. Obtenido de Secretaria Nacional de Planificaciòn y Desarrollo : http://www. planificacion.gob.ec/wp-content/uploads/downloads/2012/10/Folleto_informativo-Desconcentracion.pdf

Silva, V., Ramirez Gallegos, F., Caria, S., \& Dominguez, R. (2015). Ecuador's Buen vivir. Latin American Perspectives, 18-33.

Tungurahua, G. (mayo de 2015). Agenda Tungurahua 2015-2017. Obtenido de http://app.sni.gob.ec/sni-link/sni/PORTAL_SNI/data_sigad_plus/ sigadplusdocumentofinal/1860000130001_PDF\%20AGENDA\%20TUNGURAHUA\%202015\%20-\%202017\%20baja_30-09-2015_09-15-46.pdf

Vanhulst, J. (2015). El laberinto de los discursos del Buen vivir: entre Sumak Kawsay y Socialismo del siglo XXI. Scielo, 22.

Villena. (16 de Enero de 2017). EL ECUADOR Y EL PROCESO DE CAMBIO DE LA MATRIZ PRODUCTIVA: CONSIDERACIONES PARA EL DESARROLLO Y EQUILIBRIO DE LA BALANZA COMERCIAL. Obtenido de http://Www. eumed.net/cursecon/ecolat/ec/2015/matriz-productiva.html

Villena Izuriete, N. P. (2015). El Ecuador y el proceso de cambio de la matriz productiva: consideraciones parael desarrollo y equilibrio del a balanza comercial. Revista Observatorio de la Economia Latinoamericana, Ecuador, 4. 